\title{
Origin of the cataclysmic Late Heavy Bombardment period of the terrestrial planets
}

\author{
R. Gomes ${ }^{1,2}$, H. F. Levison ${ }^{2,3}$, K. Tsiganis ${ }^{2} \&$ A. Morbidelli ${ }^{2}$
}

The petrology record on the Moon suggests that a cataclysmic spike in the cratering rate occurred $\sim 700$ million years after the planets formed ${ }^{1}$; this event is known as the Late Heavy Bombardment (LHB). Planetary formation theories cannot naturally account for an intense period of planetesimal bombardment so late in Solar System history ${ }^{2}$. Several models have been proposed to explain a late impact spike ${ }^{3-6}$, but none of them has been set within a self-consistent framework of Solar System evolution. Here we propose that the LHB was triggered by the rapid migration of the giant planets, which occurred after a long quiescent period. During this burst of migration, the planetesimal disk outside the orbits of the planets was destabilized, causing a sudden massive delivery of planetesimals to the inner Solar System. The asteroid belt was also strongly perturbed, with these objects supplying a significant fraction of the LHB impactors in accordance with recent geochemical evidence ${ }^{7,8}$. Our model not only naturally explains the LHB, but also reproduces the observational constraints of the outer Solar System? .

Previous work ${ }^{9}$ explains the current orbital architecture of the planetary system by invoking an initially compact configuration in which Saturn's orbital period was less than twice that of Jupiter. After the dissipation of the gaseous circumsolar nebula, Jupiter's and Saturn's orbits diverged as a result of their interaction with a massive disk of planetesimals, and thus the ratio of their orbital periods, $P_{\mathrm{S}} / P_{\mathrm{J}}$, increased. When the two planets crossed their mutual 1:2 mean motion resonance $\left(1: 2 \mathrm{MMR}\right.$, that is, $\left.P_{\mathrm{S}} / P_{\mathrm{J}}=2\right)$ their orbits became eccentric. This abrupt transition temporarily destabilized the giant planets, leading to a short phase of close encounters among Saturn, Uranus and Neptune. As a result of these encounters, and of the interactions of the ice giants with the disk, Uranus and Neptune reached their current heliocentric distances and Jupiter and Saturn evolved to their current orbital eccentricities ${ }^{9}$. The main idea of this Letter is that the same planetary evolution could explain the LHB, provided that Jupiter and Saturn crossed the 1:2 MMR roughly $700 \mathrm{Myr}$ after they formed. Thus, our goal is to determine if there is a generic mechanism that could delay the migration process.

In previous studies ${ }^{9-12}$, planet migration started immediately because planetesimals were placed close enough to the planets to be violently unstable. Although this type of initial condition was reasonable for the goals of those studies, it is unlikely. Planetesimaldriven migration is probably not important for planet dynamics as long as the gaseous massive solar nebula exists. The initial conditions for the migration simulations should represent the system that existed at the time the nebula dissipated. Thus, the planetesimal disk should contain only those particles that had dynamical lifetimes longer than the lifetime of the solar nebula. In planetary systems like those we adopt from ref. 9, we find that they had to be beyond $\sim 15.3$ AU (Fig. 1), leading to the initial conditions illustrated in Fig. 2a.

In this configuration, the initial speed of migration would be
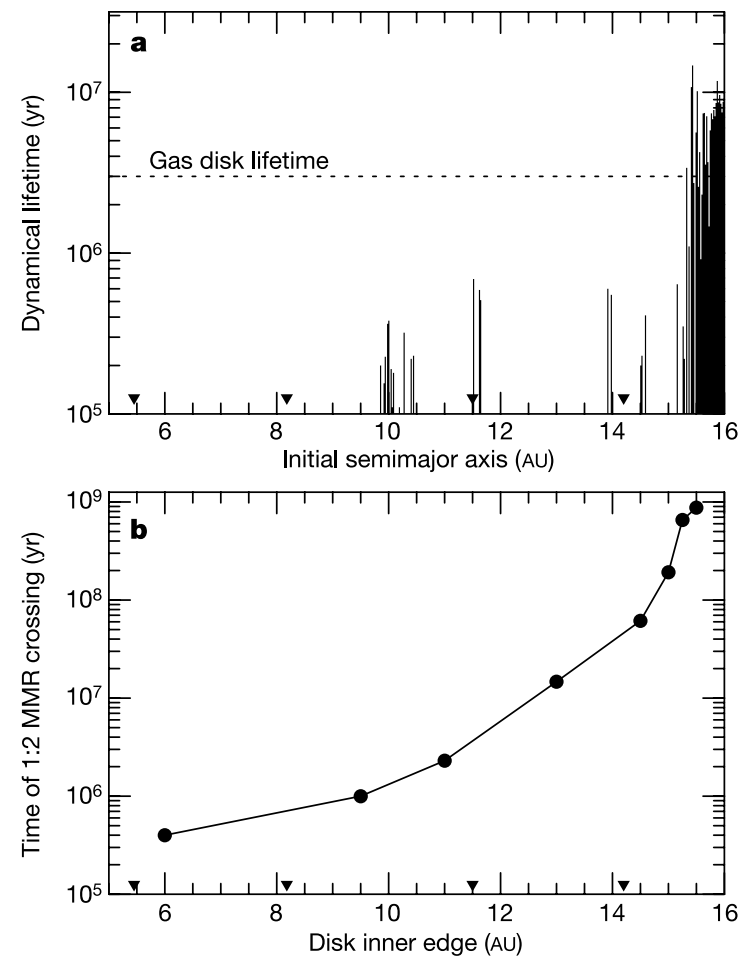

Figure 1 | Disk location and LHB timing. a, The histogram reports the average dynamical lifetime of massless test particles placed in a planetary system (shown as triangles) with Jupiter, Saturn and the ice giants on nearly circular, co-planar orbits at 5.45, 8.18, 11.5 and $14.2 \mathrm{AU}$, respectively. Initially, we placed 10 particles with $e=i=0$ (where $e$ is eccentricity and $i$ is inclination) and random mean anomaly at each semimajor axis. Stable Trojans of the planets have been removed from this computation. Each vertical bar in the plot represents the average lifetime for those 10 particles. We define 'dynamical lifetime' as the time required for a particle to encounter a planet within a Hill radius. A comparison between the histogram and the putative lifetime of the gaseous nebula ${ }^{20}$ argues that, when the latter dissipated, the inner edge of the planetesimal disk had to be about 1-1.5 AU beyond the outermost ice giant. $b$, Time at which Jupiter and Saturn crossed the 1:2 MMR, as a function of the location of the planetesimal disk's inner edge, as determined from our first set of migration simulations. In all cases, the disk had a surface density equivalent to $1.9 M_{\mathrm{E}}$ per $1 \mathrm{AU}$ annulus. The outer edge of the disk was varied so that the total mass of the disk was $35 M_{\mathrm{E}}$. The disk was initially very dynamically cold, with $e=0$ and $i<0.5^{\circ}$. A comparison between $\mathbf{a}$ and $\mathbf{b}$ shows that a disk that naturally should exist when the nebula dissipated would produce a 1:2 MMR crossing at a time comparable to that of the LHB event. 
dependent on the rate at which disk particles evolve onto planetcrossing orbits. The time at which Jupiter and Saturn cross their 1:2 MMR depends on: (1) their initial distance from the location of the resonance, (2) the surface density of the disk near its inner edge, and (3) the relative location of the inner edge of the disk and the outer ice giant. On the basis of the above arguments, we initially performed a series of eight simulations where the location of the inner edge of the disk was set as the unique free parameter (Fig. 1). As expected, we found a strong correlation between the location of the inner edge and the time of the 1:2 MMR crossing. For disks with inner edges near $15.3 \mathrm{AU}$ (see above), we find crossing times between $192 \mathrm{Myr}$ and $880 \mathrm{Myr}$ (since the beginning of the simulation).

We also performed eight simulations where we varied the initial location of the ice giants by $\sim 1 \mathrm{AU}$, Saturn's location by $\sim 0.1 \mathrm{AU}$, the total mass of the disk by 5 Earth masses $\left(5 M_{\mathrm{E}}\right)$, and its initial dynamical state by pushing the particles' eccentricities up to 0.1 and inclinations up to $3.5^{\circ}$. We found that we can delay the resonant crossing to $1.1 \mathrm{Gyr}$ since the beginning of the simulation, although longer times are clearly possible for more extreme initial conditions. Therefore, we can conclude that the global instability caused by the 1:2 MMR crossing of Jupiter and Saturn could be responsible for the LHB, because the estimated date of the LHB falls in the range of the times that we found.

Figures 2 and 3 show the evolution of one of our runs from the first series of eight. Initially, the giant planets migrated slowly owing to leakage of particles from the disk (Fig. 3a). This phase lasted $880 \mathrm{Myr}$, at which point Jupiter and Saturn crossed the 1:2 MMR. After the resonance crossing event, the orbits of the ice giants became unstable and they were scattered into the disk by Saturn. They disrupted the disk and scattered objects all over the Solar System, including the inner regions. The solid curve in Fig. 3b shows the amount of material that struck the Moon as a function of time. A total of $9 \times 10^{21} \mathrm{~g}$ struck the Moon after resonance crossing-roughly $50 \%$ of this material arrived in the first $3.7 \mathrm{Myr}$ and $90 \%$ arrived before 29 Myr. The total mass is consistent with the estimate ${ }^{4}$ of $6 \times 10^{21} \mathrm{~g}$, which was determined from the number and size distribution of lunar basins that formed around the time of the LHB epoch ${ }^{1}$. Such an influx spike happened in all our runs. The amount of cometary material delivered to the Earth is $\sim 1.8 \times 10^{23} \mathrm{~g}$, which is about $6 \%$ of the current ocean mass. This is consistent with upper bounds on the cometary contribution to the Earth's water budget, based on $\mathrm{D} / \mathrm{H}$ ratio measurement ${ }^{13}$. The average amount of material accreted by the Moon during this spike was $(8.4 \pm 0.3) \times 10^{21} \mathrm{~g}$.

The above mass delivery estimate corresponds only to the cometary contribution to the LHB, as the projectiles originated from the external massive, presumably icy, disk. However, our scheme probably also produced an in flux of material from the asteroid belt. As Jupiter and Saturn moved from 1:2 MMR towards their current positions, secular resonances (which occur when the orbit of an asteroid processes at the same rate as a planet) swept across the entire belt ${ }^{14}$. These resonances can drive asteroids onto orbit with eccentricities and inclinations large enough to allow them to evolve into the inner Solar System and hit the Moon ${ }^{4}$.

We investigated the role of asteroid impactors in our LHB model
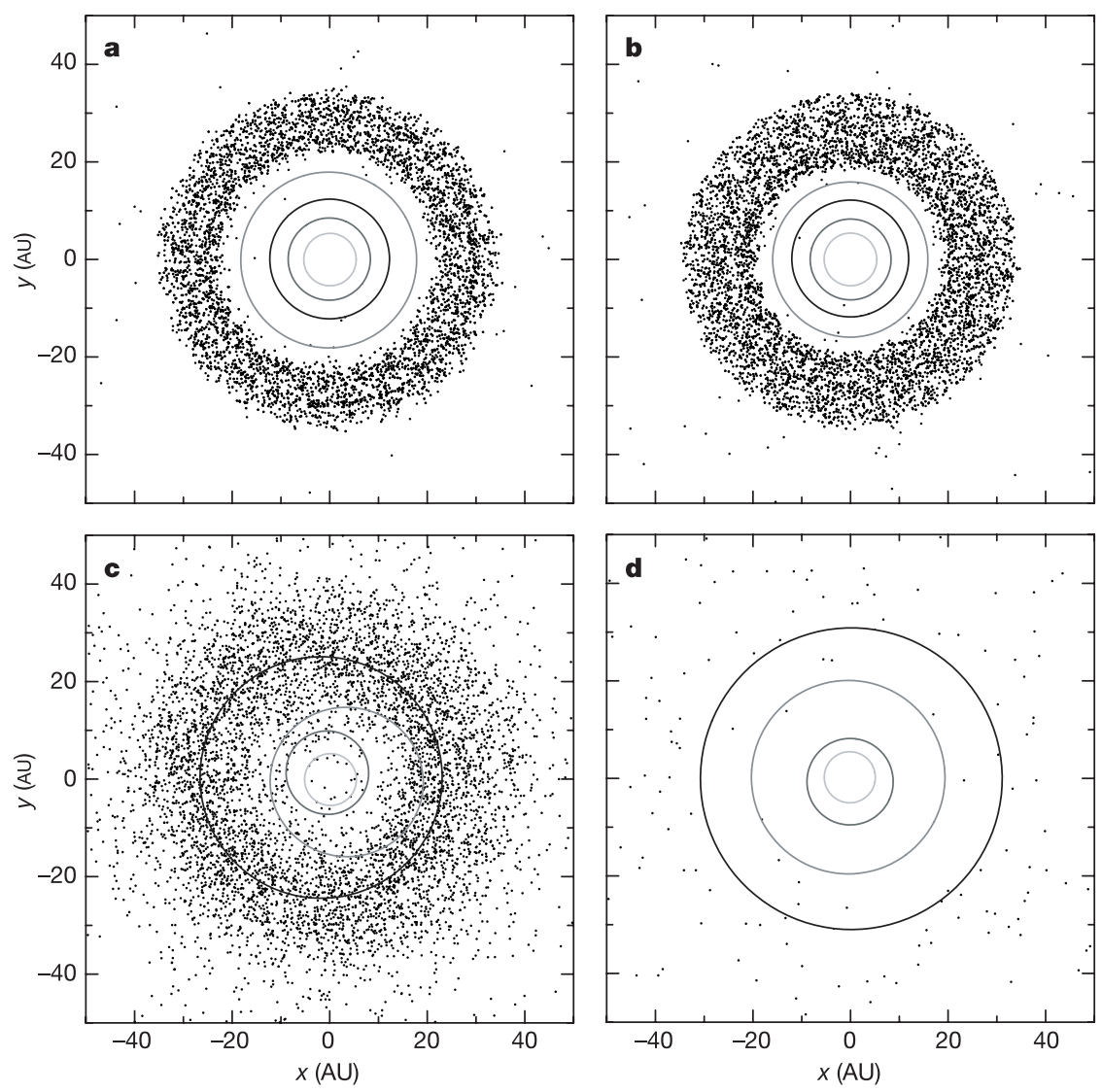

Figure 2 | The planetary orbits and the positions of the disk particles, projected on the initial mean orbital plane. The four panels correspond to four different snapshots taken from our reference simulation. In this run, the four giant planets were initially on nearly circular, co-planar orbits with semimajor axes of $5.45,8.18,11.5$ and $14.2 \mathrm{AU}$. The dynamically cold planetesimal disk was $35 M_{\mathrm{E}}$, with an inner edge at $15.5 \mathrm{AU}$ and an outer edge at $34 \mathrm{AU}$. Each panel represents the state of the planetary system at four different epochs: $\mathbf{a}$, the beginning of planetary migration (100 Myr); $\mathbf{b}$, just before the beginning of LHB ( $879 \mathrm{Myr})$; c, just after the LHB has started $(882 \mathrm{Myr})$; and d, $200 \mathrm{Myr}$ later, when only $3 \%$ of the initial mass of the disk is left and the planets have achieved their final orbits. 
by the following numerical integrations. The orbits of an asteroid belt, composed of 1,000 massless particles with semimajor axes between 2.0 and $3.5 \mathrm{AU}$, were integrated under the gravitational influence of the Sun, Venus, Earth, Mars, Jupiter and Saturn. Because formation models ${ }^{15,16}$ predict that the asteroid belt was partially depleted and dynamically excited well before the LHB, we set the particles' eccentricities between 0 and 0.3 and inclinations between $0^{\circ}$ and $30^{\circ}$, but kept the perihelion distances, $q,>1.8 \mathrm{AU}$ and aphelion distances, $Q,<4$ AU. Jupiter and Saturn were forced to migrate at rates that varied from run to run (adopted from ref. 9) by adding a suitably chosen drag-force term to their equations of motion.

We find that objects that reach Earth-crossing orbits follow one of two general paths. Some, referred to as class 1 particles, get trapped in
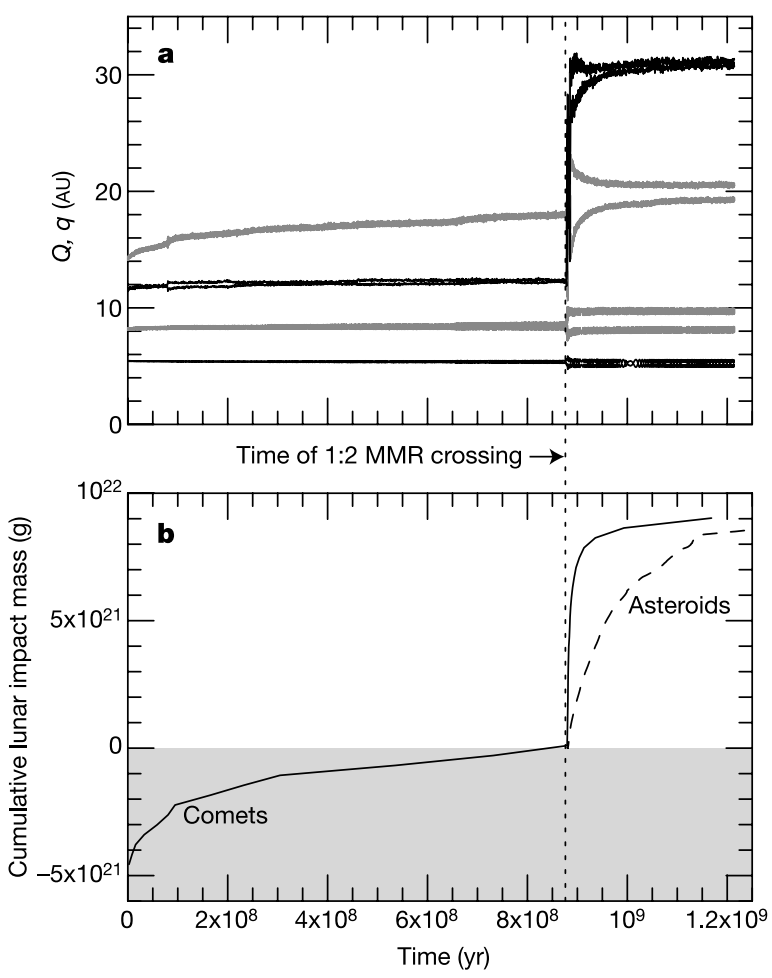

Figure 3 Planetary migration and the associated mass flux towards the inner Solar System from a representative simulation. a, The evolution of the four giant planets. Each planet is represented by a pair of curves - the top and bottom curves are the aphelion and perihelion distances, $Q$ and $q$, respectively. Jupiter and Saturn cross the 1:2 MMR at $880 \mathrm{Myr}$. The subsequent interaction between the planets and the disk led to the current planetary configuration as shown in ref. 9. b, The cumulative mass of comets (solid curve) and asteroids (dashed curve) accreted by the Moon. We have offset the comet curve so that the value is zero at the time of 1:2 MMR crossing. Thus, $\sim 5 \times 10^{21} \mathrm{~g}$ of comets was accreted before resonant crossing and $9 \times 10^{21} \mathrm{~g}$ of cometary material would have struck the Moon during the LHB. Although the terrestrial planets were not included in our cometary simulations, we estimated the amount of material accreted by the Moon directly from the mass of the planetesimal disk by combining the particles' dynamical evolution with the analytic expressions in ref. 21 . The impact velocity of these objects ranged from 10 to $36 \mathrm{~km} \mathrm{~s}^{-1}$ with an average of $21 \mathrm{~km} \mathrm{~s}^{-1}$. Estimating the asteroidal flux first requires a determination of the mass of the asteroid belt before resonant crossing. This value was determined by first combining the percentage of asteroids remaining in the belt at the end of a simulation $(\sim 10 \%$, very sensitive to planet migration rate and initial asteroid distribution) with estimates of the current mass of the belt to determine the initial asteroid belt mass $\left(\sim 5 \times 10^{-3} M_{\mathrm{E}}\right)$. The flux was then again determined by combining the particles' dynamical evolution with the analytic expressions in ref. 21 . The dashed curve shows a simulation where class 2 particles dominate. The average asteroidal impact velocity is $25 \mathrm{~km} \mathrm{~s}^{-1}$. the periapse secular resonance with Saturn (which affects eccentricities) and are driven directly onto Earth-crossing orbits. Other particles, referred to as class 2, stay in the asteroid belt, but are dynamically excited by resonant sweeping onto unstable orbits. These objects slowly leak out of the asteroid belt and can evolve into the inner Solar System. The two classes produce impact spikes with different temporal behaviours. Roughly $50 \%$ of class 1 particles arrive in the first $10 \mathrm{Myr}$, while $90 \%$ arrive within $\sim 30 \mathrm{Myr}$. Conversely, the median arrival time for class 2 particles is $\sim 50 \mathrm{Myr}$ and $90 \%$ arrive within $\sim 150$ Myr. Class 2 particles dominated in our runs (Fig. 3). However, a preliminary investigation into this issue shows that this result is probably sensitive to the exact evolution of the giant planets and the dynamical state of the asteroid belt. Thus, the best we can conclude is that the impact spike due to asteroids is between these two extremes.

We find that $(3-8) \times 10^{21} \mathrm{~g}$ of asteroids hit the Moon during our simulations (Fig. 3). This amount is comparable to the amount of comets. So, our model predicts that the LHB impactors should have been a mixture of comets and asteroids. Unfortunately, we cannot say with any certainty the exact ratio of comets to asteroids in our model because, although the amount of cometary material is fairly well constrained (probably better than a factor of 2), the amount of asteroidal material is not well known (and could be outside the range reported above), because we do not have good estimates of the mass distribution in the asteroid belt before the LHB. It should also be noted that this ratio is probably a function of impactor size, because comets and asteroids probably have different size distributions. This ratio probably also varied with time. Within the first $\sim 30$ Myr comets dominated according to these simulations, but the last impactors were asteroidal. This is consistent with recent cosmochemical findings suggesting that some of the Moon's basins were formed by asteroids $^{7,8}$.

Our results support a cataclysmic model for the lunar LHB. Although many aspects of the LHB are not well known ${ }^{1}$, our simulations reproduce two of the main characteristics attributed to this episode: (1) the $700 \mathrm{Myr}$ delay between the LHB and terrestrial planet formation, and (2) the overall intensity of lunar impacts. Our model predicts a sharp increase in the impact rate at the beginning of the LHB. Unfortunately, the available lunar data are not yet capable of addressing this prediction.

Our model also has the advantage of supplying impactors that are a mixture of comets and asteroids. Our model predicts that the asteroid belt was depleted by a factor of $\sim 10$ during the LHB. This depletion does not contradict collisional evolution models ${ }^{17,18}$. On the contrary, the late secular resonance sweeping could explain why we do not see a large number of asteroid families that were produced during the $\mathrm{LHB}^{18}$. Our model predicts that the LHB lasted from between $\sim 10$ Myr and $\sim 150$ Myr. Correspondingly, the drop-off in impact rates could be quite fast (with $50 \%$ of the impacts occurring in the first $3.7 \mathrm{Myr}$ and $90 \%$ in $29 \mathrm{Myr}$ ) or moderately slow (with $50 \%$ of the impacts occurring in the first $50 \mathrm{Myr}$ and $90 \%$ in $150 \mathrm{Myr}$ ) We are unable to pinpoint more exact values because the duration and the drop-off of the LHB depends on the relative contributions of class 1 asteroids, class 2 asteroids, and comets, which in turn are very sensitive to the pre-LHB orbital structure of the asteroid belt.

Most importantly, our scheme for the LHB is the result of a generic migration-delaying mechanism, followed by an instability, which is itself induced by a deterministic mechanism of orbital excitation of the planets ${ }^{9}$.This revised planetary migration scheme naturally accounts for the currently observed planetary orbits ${ }^{9}$, the LHB, the present orbital distribution of the main-belt asteroids and the origin of Jupiter's Trojans ${ }^{19}$.

\section{Received 6 December 2004; accepted 18 April 2005.}

1. Hartmann, W. K., Ryder, G., Dones, L. \& Grinspoon, D. in Origin of the Earth and Moon (eds Canup, R. \& Righter, K.) 493-512 (Univ. Arizona Press, Tucson, 2000). 
2. Morbidelli, A., Petit, J.-M., Gladman, B. \& Chambers, J. A plausible cause of the Late Heavy Bombardment. Meteorit. Planet. Sci. 36, 371-380 (2001).

3. Zappala, V., Cellino, A., Gladman, B. J., Manley, S. \& Migliorini, F. Asteroid showers on Earth after family break-up events. Icarus 134, 176-179 (1998).

4. Levison, H. F. et al. Could the lunar "Late Heavy Bombardment" have been triggered by the formation of Uranus and Neptune? Icarus 151, 286-306 (2001).

5. Chambers, J. E. \& Lissauer, J. J. A new dynamical model for the lunar Late Heavy Bombardment. Lunar Planet. Sci. Conf. XXXIII, abstr. 1093 (2002)

6. Levison, H. F., Thommes, E. W., Duncan, M. J., Dones, L. A. in Debris Disks and the Formation of Planets: A Symposium in Memory of Fred Gillett (11-13 April 2002, Tucson, Arizona) (eds Caroff, L., Moon, L. J., Backman, D. \& Praton, E.) 152-167 (ASP Conf. Ser. 324, Astronomical Society of the Pacific, San Francisco, 2005).

7. Kring, D. A. \& Cohen, B. A. Cataclysmic bombardment throughout the inner Solar System 3.9-4.0 Ga. J. Geophys. Res. Planets 107(E2), 4-10 (2002).

8. Tagle, R. LL-ordinary chondrite impact on the Moon: Results from the $3.9 \mathrm{Ga}$ impact melt at the landing site of Apollo 17. Lunar Planet. Sci. Conf. XXXVI, abstr. 2008 (2005)

9. Tsiganis, K., Gomes, R., Morbidelli, A. \& Levison, H. F. Origin of the orbital architecture of the giant planets of the Solar System. Nature doi:10.1038/ nature03539 (this issue).

10. Fernandez, J. A. \& Ip, W.-H. Some dynamical aspects of the accretion of Uranus and Neptune-The exchange of orbital angular momentum with planetesimals. Icarus 58, 109-120 (1984).

11. Hahn, J. M. \& Malhotra, R. Orbital evolution of planets embedded in a planetesimal disk. Astron. J. 117, 3041-3053 (1999)

12. Gomes, R. S., Morbidelli, A. \& Levison, H. F. Planetary migration in a planetesimal disk: Why did Neptune stop at 30 AU? Icarus 170, 492-507 (2004).

13. Morbidelli, A. et al. Source regions and timescales for the delivery of water to
Earth. Meteorit. Planet. Sci. 35, 1309-1320 (2000)

14. Gomes, R. S. Dynamical effects of planetary migration on the primordial asteroid belt. Astron. J. 114, 396-401 (1997)

15. Wetherill, G. W. An alternative model for the formation of the asteroids. Icarus 100, 307-325 (1992)

16. Petit, J., Morbidelli, A. \& Chambers, J. The primordial excitation and clearing of the asteroid belt. Icarus 153, 338-347 (2001).

17. Davis, D. R., Ryan, E. V. \& Farinella, P. Asteroid collisional evolution: results from current scaling algorithms. Planet. Space Sci. 42, 599-610 (1994).

18. Bottke, W. et al. The fossilized size distribution of the main asteroid belt. Icarus 175(1), 111-140 (2005).

19. Morbidelli, A., Levison, H. F., Tsiganis, K. \& Gomes, R. Chaotic capture of Jupiter's Trojan asteroids in the early Solar System. Nature doi:10.1038/ nature 03540 (this issue)

20. Haisch, K. E., Lada, E. A. \& Lada, C. J. Disk frequencies and lifetimes in young clusters. Astrophys. J. 553, L153-L156 (2001).

21. Wetherill, G. W. Collisions in the asteroid belt. J. Geophys. Res. 72, 2429-2444 (1967).

Acknowledgements R.G. thanks Conselho Nacional de Desenvolvimento Científico e Tecnológico for support for his sabbatical year in the OCA observatory in Nice. K.T. was supported by an EC Marie Curie Individual Fellowship. A.M. and H.F.L. thank the CNRS and the NSF for funding collaboration between the OCA and the SWRI groups. H.F.L. was supported by NASA's Origins and PG\&G programmes.

Author Information Reprints and permissions information is available at npg.nature.com/reprintsandpermissions. The authors declare no competing financial interests. Correspondence and requests for materials should be addressed to A.M. (morby@obs-nice.fr). 